High-resolution 3-D imaging of surface damage sites in fused silica with Optical Coherence Tomography

G. Guss, I. Bass, R. Hackel, C. Mailhiot, S. G. Demos

November 6, 2007

SPIE - Boulder Damage Symposium Boulder, CO, United States September 24, 2007 through September 26, 2007 
This document was prepared as an account of work sponsored by an agency of the United States government. Neither the United States government nor Lawrence Livermore National Security, LLC, nor any of their employees makes any warranty, expressed or implied, or assumes any legal liability or responsibility for the accuracy, completeness, or usefulness of any information, apparatus, product, or process disclosed, or represents that its use would not infringe privately owned rights. Reference herein to any specific commercial product, process, or service by trade name, trademark, manufacturer, or otherwise does not necessarily constitute or imply its endorsement, recommendation, or favoring by the United States government or Lawrence Livermore National Security, LLC. The views and opinions of authors expressed herein do not necessarily state or reflect those of the United States government or Lawrence Livermore National Security, LLC, and shall not be used for advertising or product endorsement purposes. 


\title{
High-resolution 3-D imaging of surface damage sites in fused silica with Optical Coherence Tomography
}

\author{
Gabe Guss*, Isaac Bass, Richard Hackel, Christian Mailhiot, Stavros G. Demos \\ Lawrence Livermore National Laboratory, 7000 East Ave., Livermore, CA, USA 94550
}

\begin{abstract}
In this work, we present the first successful demonstration of a non-contact technique to precisely measure the 3D spatial characteristics of laser induced surface damage sites in fused silica for large aperture laser systems by employing Optical Coherence Tomography (OCT). What makes OCT particularly interesting in the characterization of optical materials for large aperture laser systems is that its axial resolution can be maintained with working distances greater than $5 \mathrm{~cm}$, whether viewing through air or through the bulk of thick optics. Specifically, when mitigating surface damage sites against further growth by $\mathrm{CO}_{2}$ laser evaporation of the damage, it is important to know the depth of subsurface cracks below the damage site. These cracks are typically obscured by the damage rubble when imaged from above the surface. The results to date clearly demonstrate that OCT is a unique and valuable tool for characterizing damage sites before and after the mitigation process. We also demonstrated its utility as an in-situ diagnostic to guide and optimize our process when mitigating surface damage sites on large, high-value optics.
\end{abstract}

Keywords: optical coherence tomography, fused silica, surface damage

\section{INTRODUCTION}

The laser system being built at the National Ignition Facility (NIF) utilizes 192 fused silica lenses to focus $351 \mathrm{~nm}$ light onto a deuterium-tritium fusion target. The thicknesses of the lenses can vary from $18 \mathrm{~mm}$ to as much as $54 \mathrm{~mm}$ over a $40 \mathrm{~cm}$ rectangular aperture. These lenses will be exposed to mean fluences as high as $8 \mathrm{~J} / \mathrm{cm}^{2}$ and local peak fluences of $>14 \mathrm{~J} / \mathrm{cm}^{2}$. Surface damage in fused silica is known to initiate in this fluence range. ${ }^{1}$ Once initiated, damage will grow exponentially, in diameter and depth, upon further exposure. ${ }^{2}$

Many techniques have been developed to mitigate the growth of damage, extending the lifetime and operability of highvalue fused silica optics. ${ }^{3}$ The most effective technique has been removal of the damage by a combination of melting and ablation produced by a tightly focused $10.6 \mu \mathrm{m}$ beam from a $\mathrm{CO}_{2}$ laser. Mitigation of damage sites as large as 300 $\mu \mathrm{m}$ in diameter with cracks $150 \mu \mathrm{m}$ deep were successfully mitigated using fast-scanning galvanometers to move the $10.6 \mu \mathrm{m}$ laser beam in a spiral pattern over the damage site. ${ }^{4}$ Mitigation by this technique was observed to be almost entirely ablative. That is, successful mitigation required removal of material to the deepest cracks.

Development of new protocols to mitigate damage sites larger than $300 \mu \mathrm{m}$ in diameter has been hindered by a lack of information about damage site subsurface cracks. One technique for studying subsurface features is to use a conventional microscope to view a damage site artificially created on a specially prepared thin slice of fused silica. The microscope is focused along the thin direction of the sample to obtain a side view of the damage site. The working distance of a conventional microscope makes this measurement impossible on the large aperture NIF focusing lenses.

The geometry of surface damage on large aperture lenses further complicates the problem of optical inspection. If the inspection instrument is oriented to measure the subsurface cracks from the surface of the optic on which the damage is located, then the probe light must penetrate through the finely grained silica rubble which forms the damage pit. The light scattering properties of this rubble prevents front side viewing using techniques such as laser scanning confocal microscopy.

Accurate profiling of subsurface cracks can be accomplished by back side viewing of the surface damage through the bulk of the silica. For thick NIF focusing lenses, the optical inspection instrument is required to have a minimum

* guss2@1lnl.gov; phone 1925 422-3288 
working distance of $5 \mathrm{~cm}$. In order to accurately choose ablation mitigation parameters, the resolution of the damage site profiles needs to be less than $25 \mu \mathrm{m}$. Resolutions larger than this may still yield information valuable to mitigation, but at the expense of inexact ablation protocols.

Optical coherence tomography is a 3D profiling technique used primarily in medical imaging. It provides cross sectional images of tissues and media which are partially opaque. It has found application in many clinical specialties, including intravascular and retinal imaging. Cross sectional imaging of laser damage using OCT has been previously reported. ${ }^{5}$

This paper describes the use of an OCT instrument ${ }^{6}$ for the purpose of 3D profiling surface damage sites in fused silica. The OCT is found to meet the profiling requirements for the purpose of mitigating thick NIF focusing lenses. The layout of the instrument, its characteristics and operation will be reported. Data analysis which leads to accurate 3D profiles will be discussed. Experimental results will show that the OCT profiles compare accurately with side-view microscopy.

\section{OCT INSTRUMENT CHARACTERISTICS AND OPERATION}

A schematic diagram of the OCT instrument chosen for its long working distance is shown in Fig 1. The important components of the interferometer design are: a wide bandwidth super luminescent diode light source, a variable, highspeed optical path length (OPL) scanning device, a fiber coupled measurement probe and a photodiode detector with associated electronic filtering, data acquisition and digital signal processing (DSP).

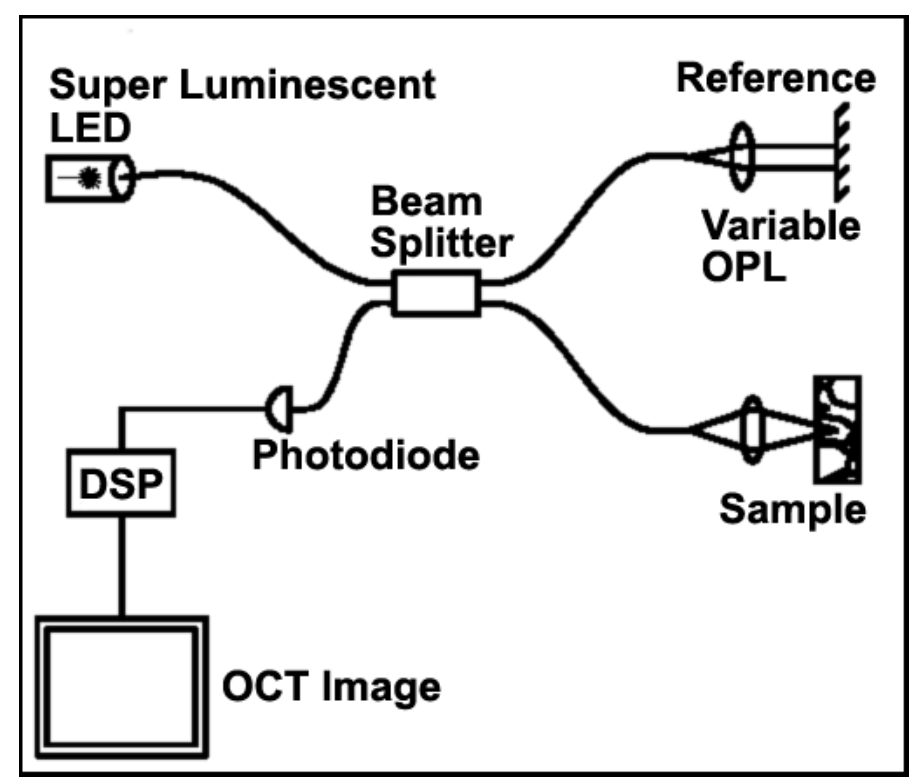

Figure 1: Schematic layout of OCT instrument.

Interference fringes occur when the optical path length (OPL) of light traveling in the reference and sample legs of the interferometer are nearly the same. The bandwidth of the super luminescent diode light source determines the OPL range over which interference can occur. For an $80 \mathrm{~nm}$ bandwidth diode, the full-width-half-max (FWHM) of the interference peak is measured to be $20 \mu \mathrm{m}$. Artifacts inherent to the OCT system generate interference side-lobes with signal levels of the order of $1 \%$ of the peak interference signal at distances extending $100 \mu \mathrm{m}$ from the peak. Side-lobes from bright features have the potential of masking the signal from weaker features (see Fig. 2). Data analysis techniques must therefore be developed to filter these side-lobes in order to achieve the required signal sensitivity. 


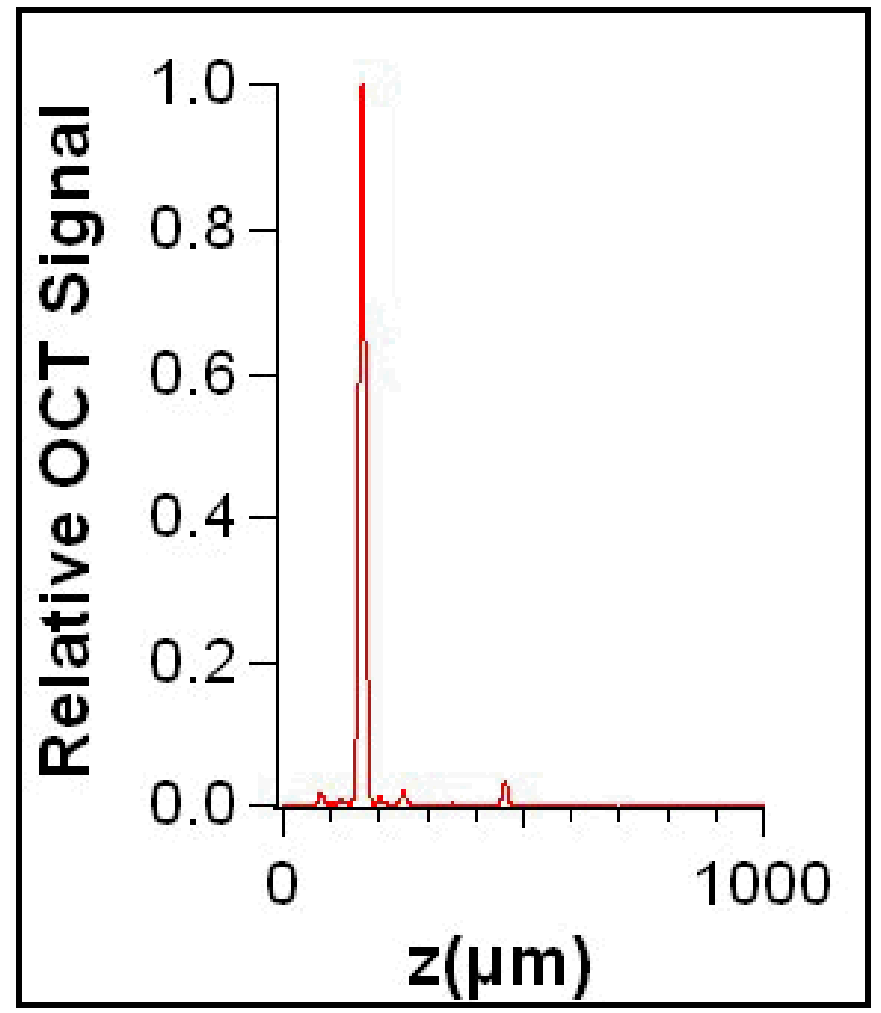

Figure 2. OCT interference signal from the surface and a subsurface feature of a fused silica sample. The FWHM of interference peak is $20 \mu \mathrm{m}$, but low level artifacts extend to $100 \mu \mathrm{m}$ on each side of the peak.

A unique optical device constructed out of rhombic prisms scans the $\mathrm{OPL}^{7}$ (see Fig. 3). It allows rapid collection of data at a rate of 300 axial scan-lines per second. Each line consists of the interference signal as a function of distance along the optical axis of the sample probe lens (Fig. 2). The data collection system uses a photodiode to sample the optical signal at $1 \mu \mathrm{m}$ intervals over a distance of $4 \mathrm{~mm}$ surrounding the focus point of the lens. The beginning of the collection region can be adjusted to measure a region of interest.

The lateral resolution of the instrument is determined by the diameter of the spot generated by the focus lens. This diameter varies from 25 to $30 \mu \mathrm{m}$ over the 4-mm axial region of interest when the focus spot is centered on this region. Three-dimensional profiles are obtained by simultaneously scanning the probe lens in $\mathrm{x}$ and $\mathrm{y}$ with a pair of translation stages. Axial data lines are collected as the probe scans in $\mathrm{x}$, thus producing an $\mathrm{x}-\mathrm{z}$ cross-sectional image (see Fig. 4). The lens then moves one step in $\mathrm{y}$ and the $\mathrm{x}$ scan is repeated. The collection of cross sectional images results in a volume measurement.

The overall process time depends on many factors. Two main parameters are easily adjusted: the size of the region of interest and the number of measurements made in each dimension. The scans presented here have a $2 \mathrm{~mm} \times 2 \mathrm{~mm} \times$ $1 \mathrm{~mm}$ region of interest, with data collected at $1 \mu \mathrm{m}$ intervals in all three dimensions. This results in 4 billion measurements and takes 2 hours to collect. 


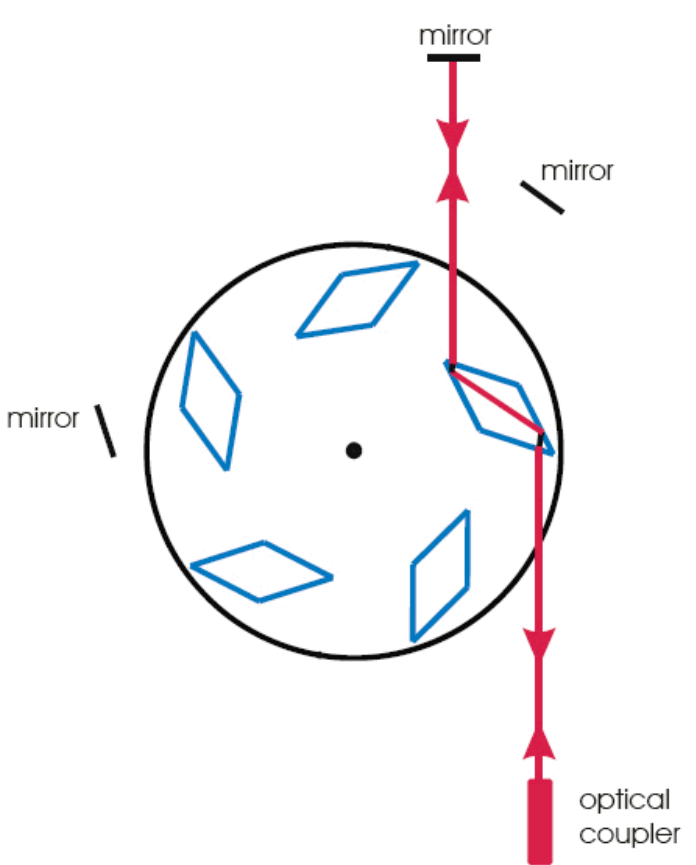

Direct

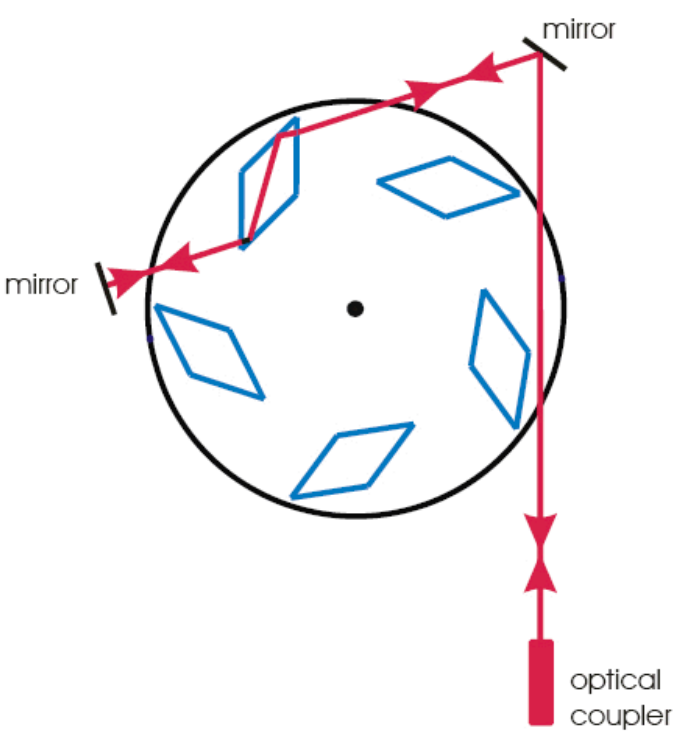

Reinjection

Figure 3 shows a unique optical device constructed with rhombic prisms. As the prisms rotate, the optical path length of the reference path of the interferometer is varied by $4 \mathrm{~mm}$ in air. (Image courtesy of Novacam Technologies, Inc. ${ }^{7}$ )

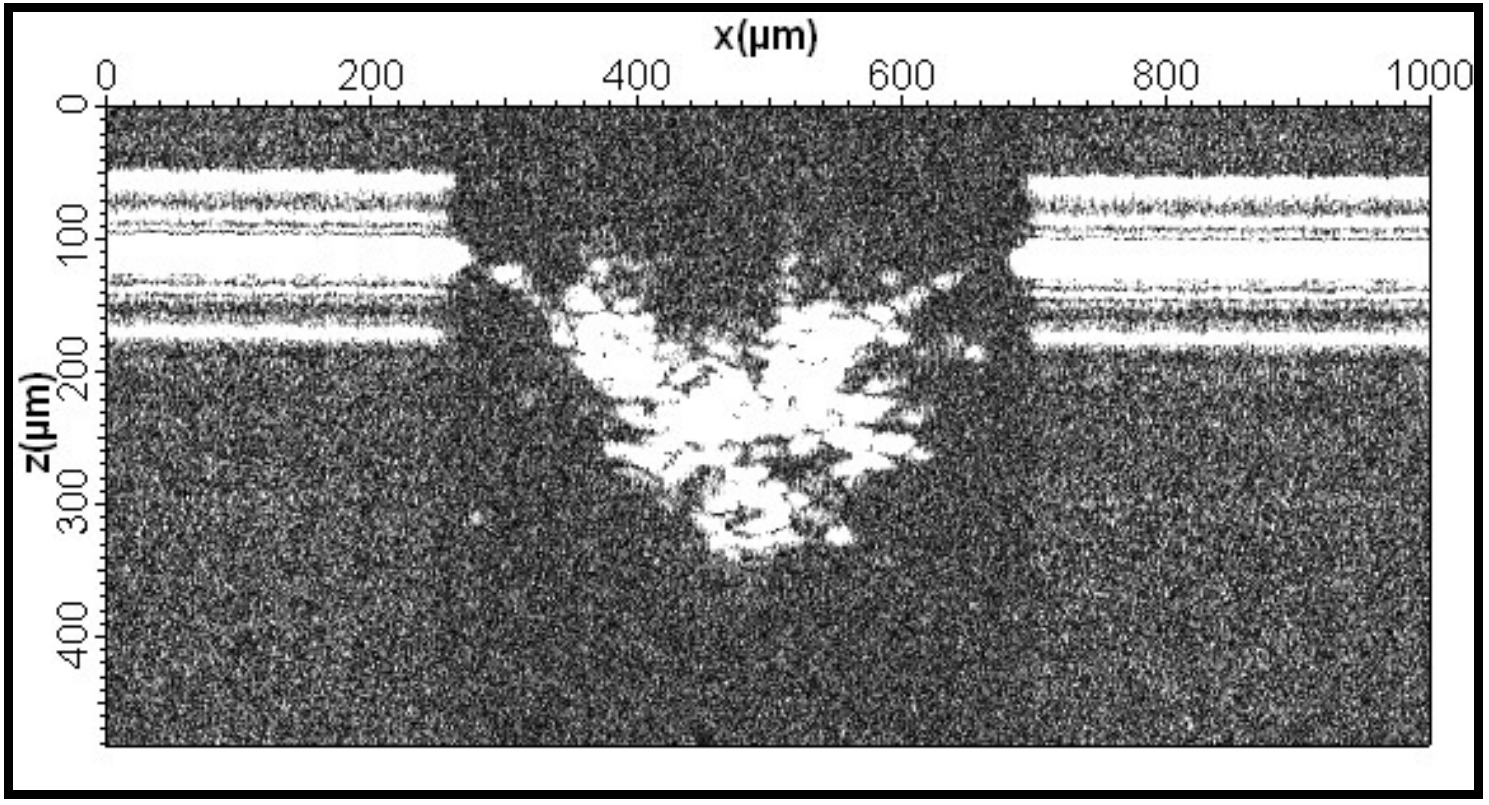

Figure 4 is an image of a single cross-sectional plane of a surface damage site in fused silica. The image contrast is adjusted to illustrate the signal-to-noise level and side-lobe artifacts surrounding the signal from the sample surface. 


\section{ANALYSIS OF CROSS SECTIONAL IMAGES RESULTS IN A 3D IMAGE}

Our analysis technique reduces the signal from a cross-sectional image to a set of point locations. Each point is approximately a $25 \mu \mathrm{m}$ square and corresponds to an important feature in the measurement plane. The features of interest are damaged material, and subsurface cracks which scatter or reflect light into the interferometer. The location of the undamaged surface relative to the damaged material is also of interest. Each feature scanned by the OCT generates an interference signal which differentiates it from the undamaged material.

The side-lobe pattern of the OCT signal from a feature was calibrated using the signal generated by the polished surface of a fused silica sample (see Fig. 5). A family of calibration curves as a function of the peak amplitude was obtained by tilting the sample to vary the amplitude.

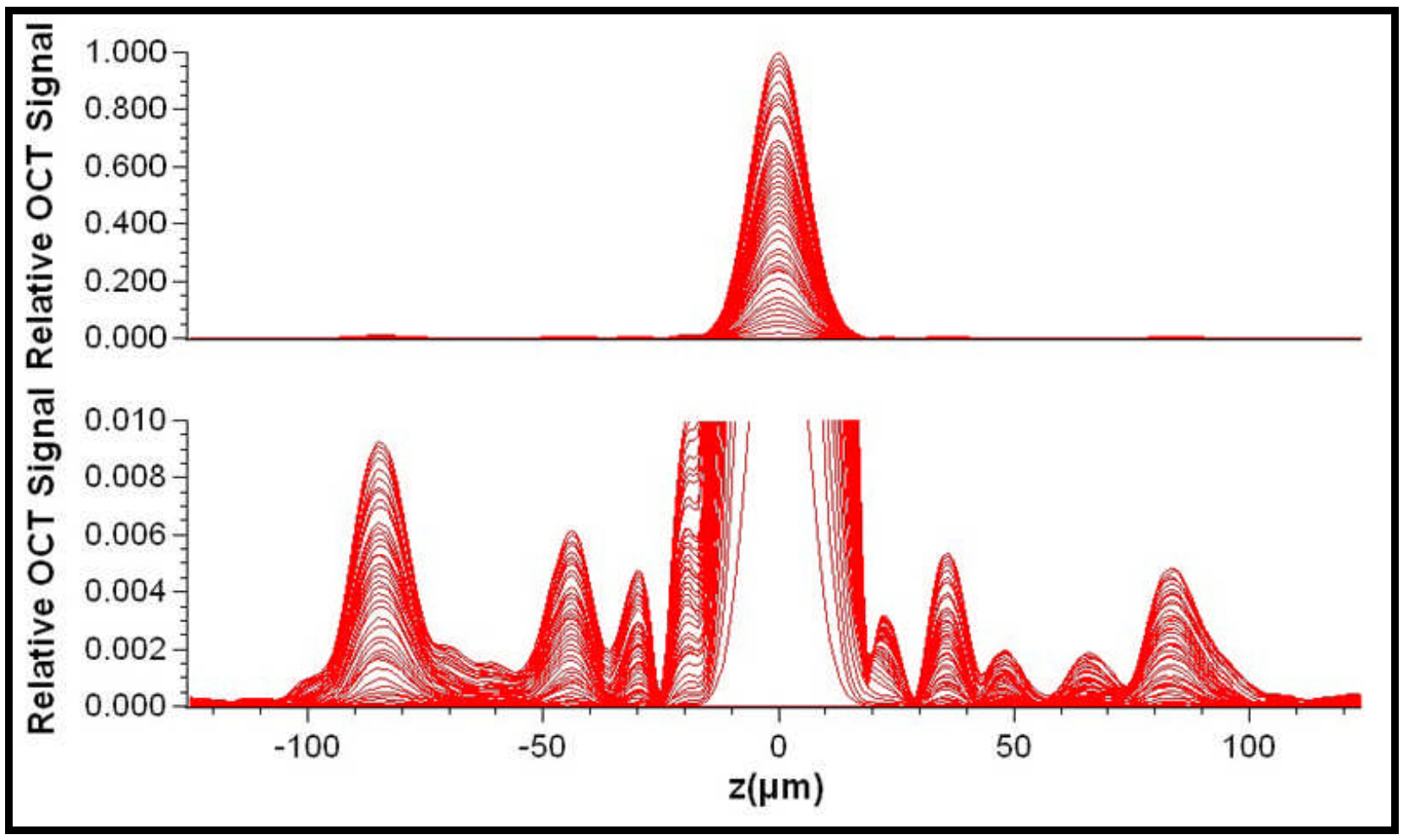

Figure 5 shows a family of calibration curves for the OCT signal from features of a sample with different scattering properties. These curves were obtained from the signal produced by the polished surface of a fused-silica sample.

This family of calibration curves was used to recover the point locations of the features from the raw intensity data. For any particular $\mathrm{x}-\mathrm{y}$ location on the sample, the recorded axial intensity as a function of position along a vertical line through that location in the cross-sectional image, is the sum of the OCT calibration signals corresponding to features on that line. The range of signal amplitudes of the features is large enough to cause the side lobes of one to have an amplitude equal to or greater than the peak amplitude of another feature. This prevents simply converting the peak locations into point data.

A simple algorithm was implemented in the data analysis to convert the signal from each vertical line in a crosssectional image into a list of point locations (see Fig. 6). The algorithm begins by finding the location of the largest peak in the line. The x-y-z location of this peak is saved. The intensity of the peak is then used to pick the correct calibration curve, which includes the main peak and side lobes. The calibration curve is registered to the peak in the line and subtracted from the data, thus reducing the signal from that feature below the background level of the line. This process is repeated for the next largest amplitude peak and so-on, iteratively until all peaks are below a threshold value. This threshold level is determined by measuring the standard deviation of the background signal for each line in a region which is known to have no features. The threshold value is set to 6 times the measured sigma. 


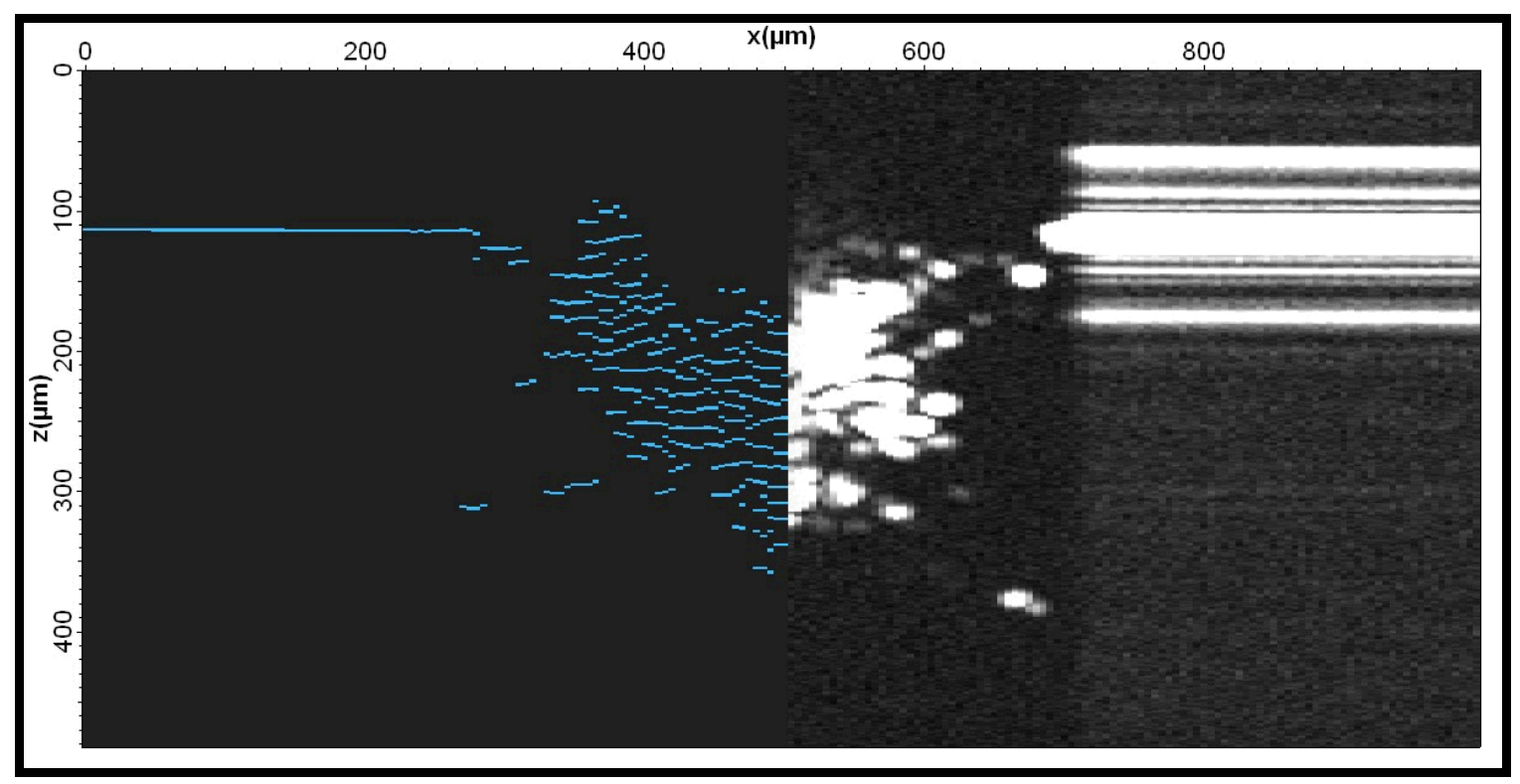

Figure 6 illustrates the effect of applying the feature location algorithm to a cross sectional image. The vertical lines to the left of the $500 \mu \mathrm{m} x$-axis position have been reduced to binary data, each point corresponding to a feature.

\section{COMPARISON TO MICROSCOPY VERIFIES ACCURACY OF OCT PROFILING OF DAMAGE SITES}

We evaluated the accuracy of the 3D profiles of subsurface damage measured from the backside with OCT by comparing them with measurements of the same damage made with conventional microscopy. This was accomplished by generating the damage on a thin strip of fused silica that allowed conventional microscopy from two orthogonal directions. The usual longitudinal direction coupled with the side-view direction gave information about the shape and morphology of both surface damage and subsurface cracks (see Fig. 7).

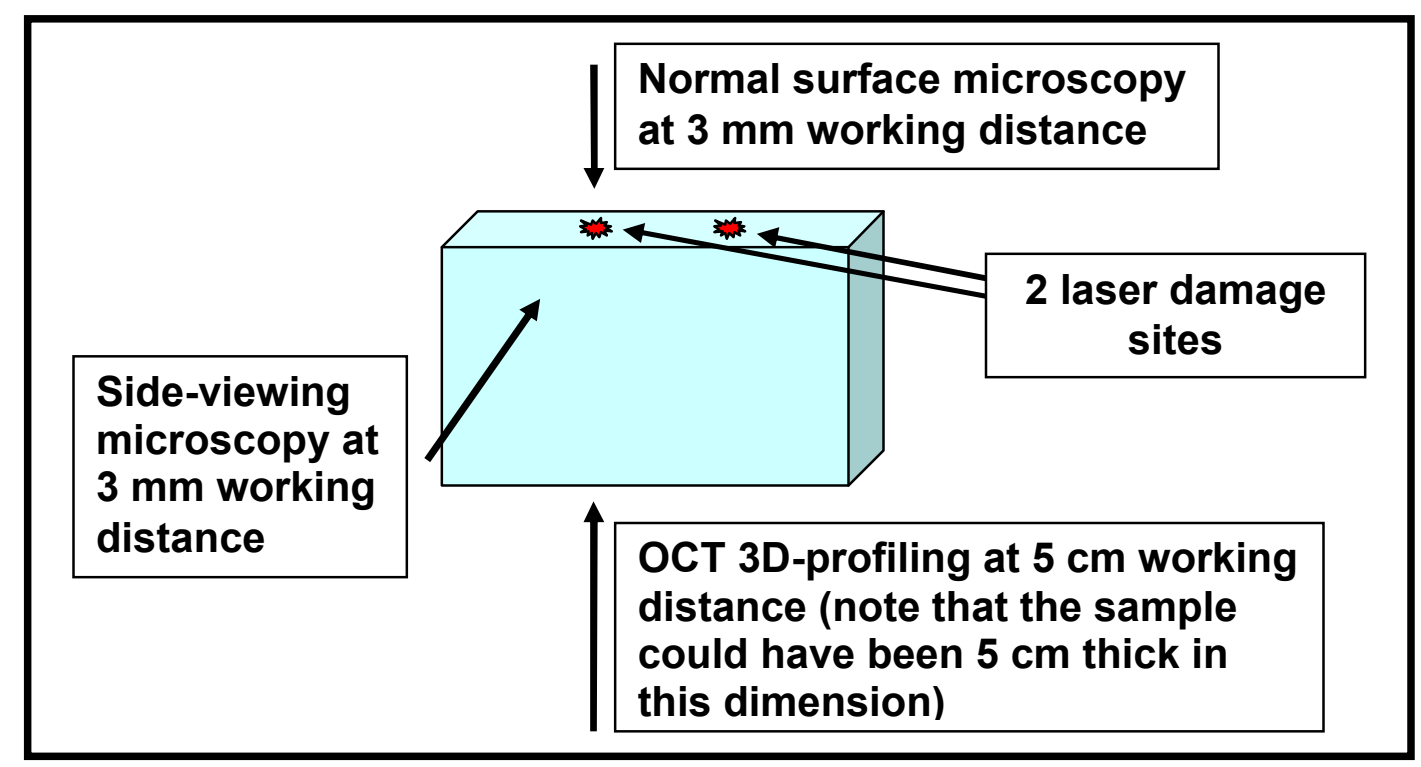

Figure 7 shows the experimental setup for verifying the accuracy of OCT profiling. The OCT scan is performed from the backside of the optic at a distance of $5 \mathrm{~cm}$ and compared with front and side view microscopy at $3 \mathrm{~mm}$ working distance. 
Damage was generated by focusing a $355 \mathrm{~nm}$ wavelength pulse of light on the surface of the fused silica. The spot size was $75 \mathrm{um}$ with a fluence which varied between 50 and $200 \mathrm{~J} / \mathrm{cm}^{2}$. This process creates damage which has varied amounts of subsurface cracking suitable for comparing OCT and microscopy.

The OCT scans of the damage sites were acquired from the back side at a working distance of $5 \mathrm{~cm}$. The transverse area covered was a 2-mm square, and the axial region of interest was $1 \mathrm{~mm}$ long extending from just above the surface to well below the damage site features. The scans collected data at $1 \mu \mathrm{m}$ step size in all three dimensions. In order to improve signal to noise levels of the faintest subsurface features, the data was averaged into $5 \mu \mathrm{m}$ bins in the transverse direction. The microscope images were collected with a standard 10x objective and scaled using a calibration reticule. The OCT data was rotated and plotted to match the viewing direction of the microscope.

The comparison between the standard longitudinal microscope image is visually comparable to the projection of the OCT data onto the xy-plane (see Figs. 8 and 9). The crack starting in the lower left of the microscope image at (-200, 200 ) is seen from the OCT data to extend from the surface downward into the bulk toward the center of the damage. This type of crack is known to be shallow, and the OCT data reveals that it is located in the 0 to $50 \mu \mathrm{m}$ depth region. The radial crack extending in the 2 o'clock direction at $(175,200)$ is located at a depth of $200 \mu \mathrm{m}$ in the OCT image. The depth of this crack is impossible to determine from the microscope image.

The comparison between the side view microscope image of the damage site is comparable to the OCT data projected along the same viewing direction. The deepest crack is $300 \mu \mathrm{m}$ below the surface and is located at the same transverse position in both the microscope image and the OCT plot. The crack which occurs at a depth of $250 \mu \mathrm{m}$ and at a transverse location of $200 \mu \mathrm{m}$ in the microscope image, appears to be two cracks in the OCT plot. This is due to blurring caused by one of the overlapping cracks being out of the microscopes focus plane. The OCT data allows the study of features which are out of focus or overlapping in traditional microscopy.

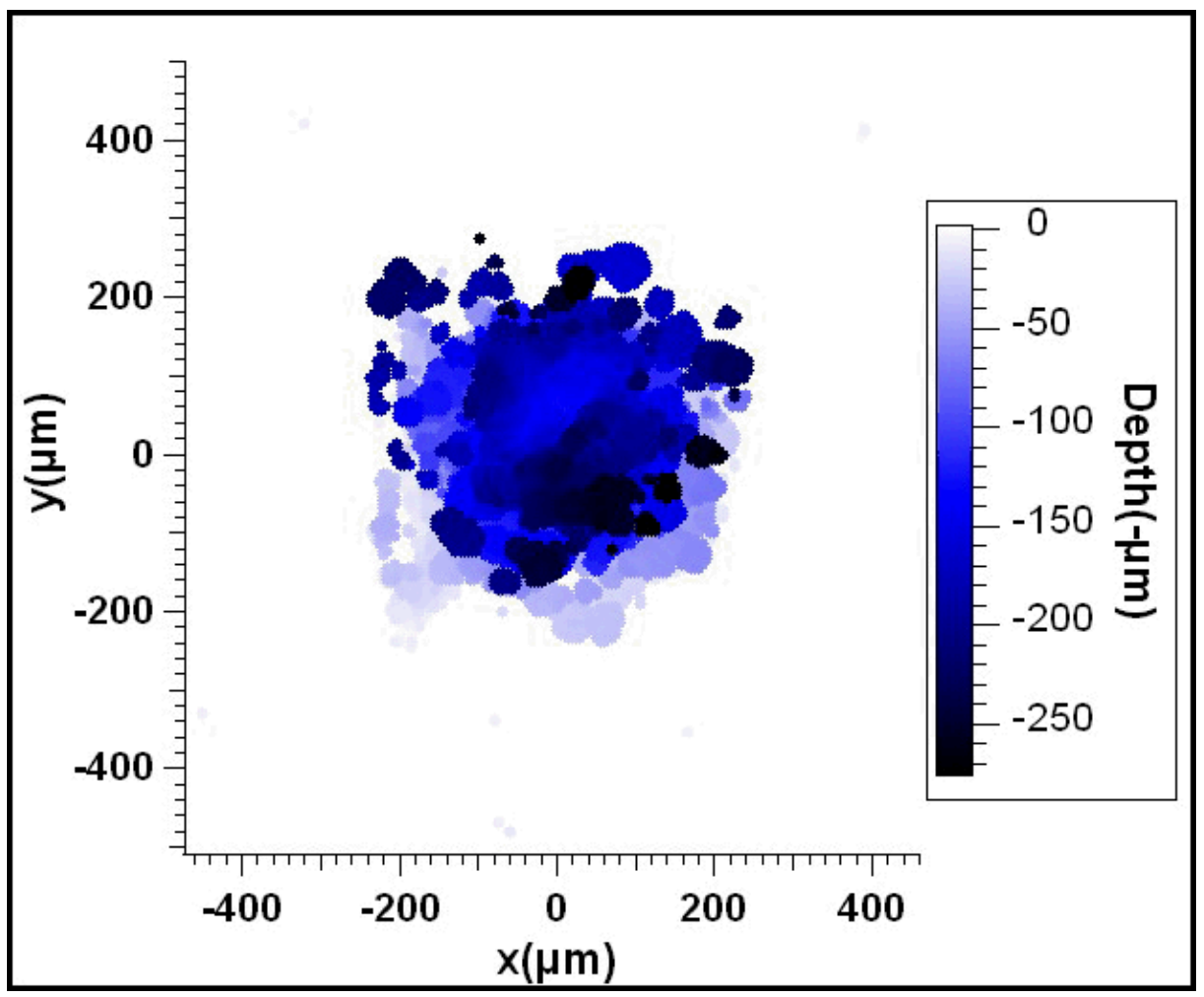

Figure 8. OCT data projected longitudinally onto the surface with depth information indicated by the color scale. 


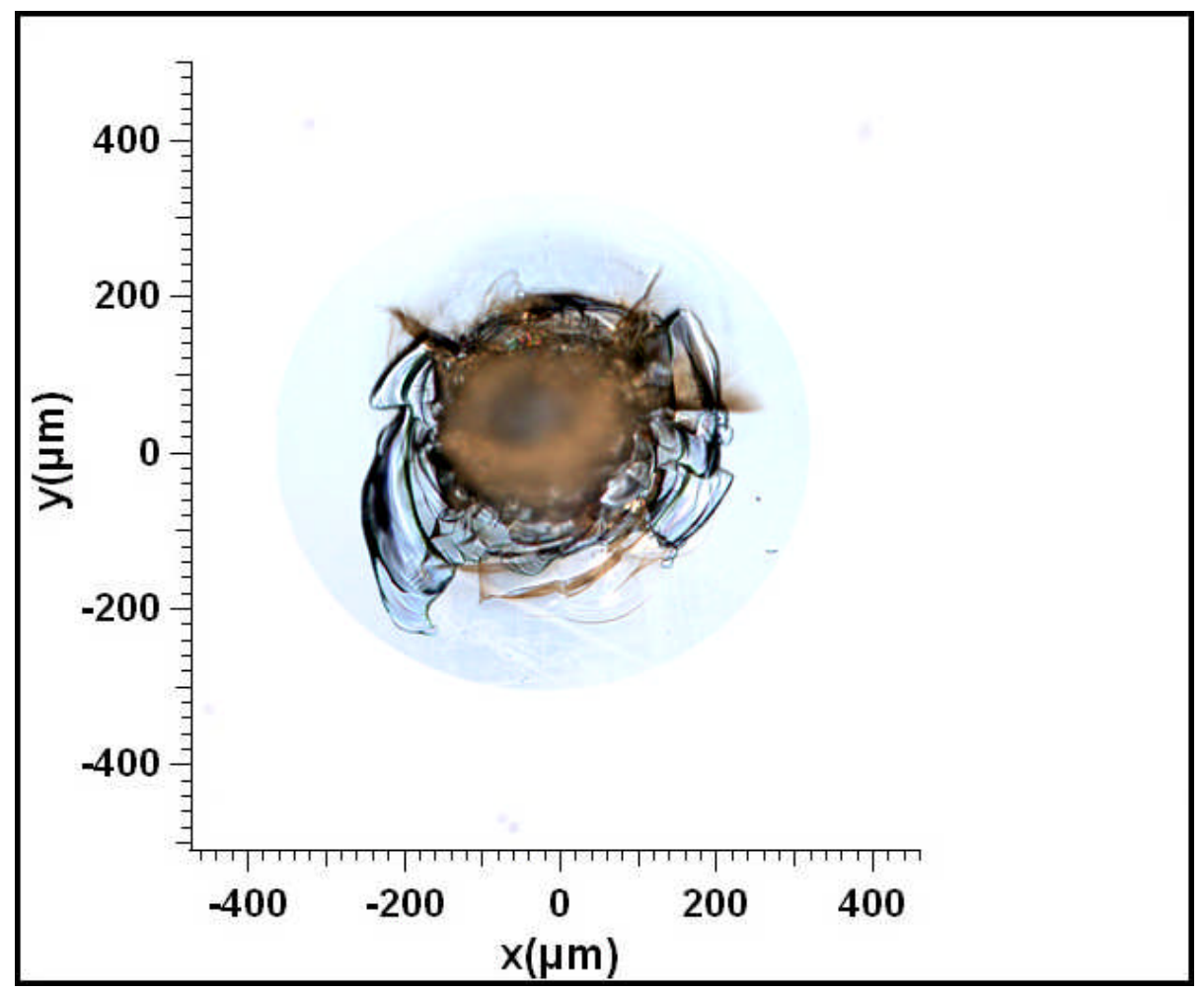

Figure 9. Longitudinal microscope image of the damage site shown in figure 8.

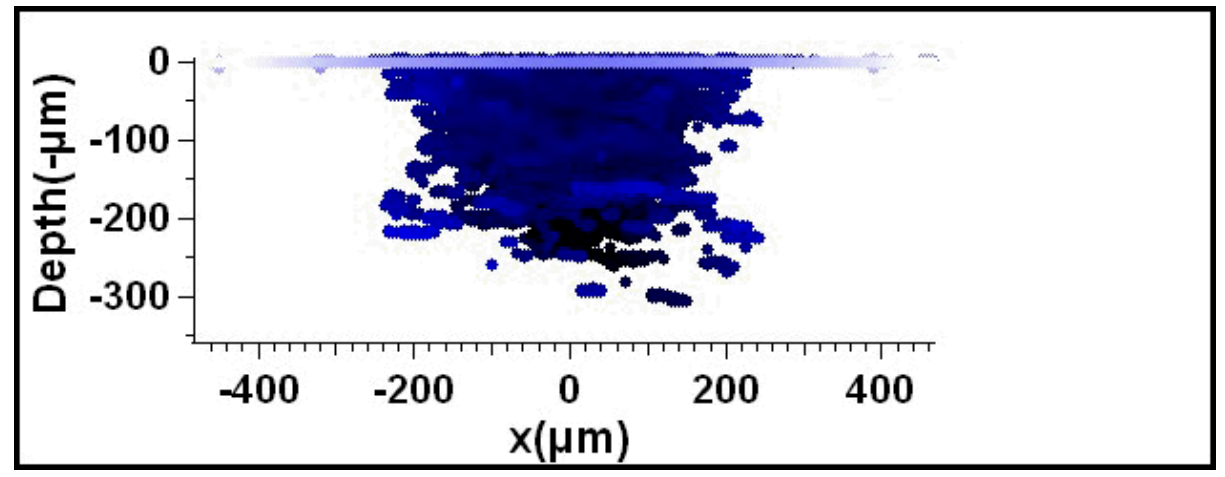

Figure 10. OCT data projected along the same direction as used for the microcope side-view image.

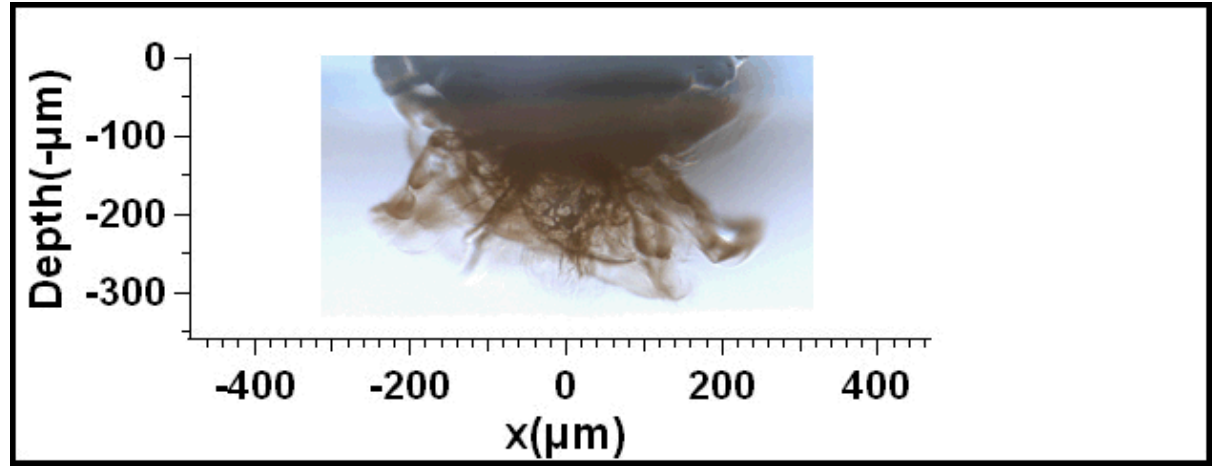

Figure 11. Side view microscope image of the damage site. 


\section{SUMMARY}

OCT is a powerful diagnostic useful for studying damage in fused silica. It can provide full 3D profiling of the features of a damage site. Mitigation of damage, a topic of importance at NIF, is becoming more dependent on knowledge of the morphology of the damage and subsurface cracking. The optics of interest at NIF have large apertures and are thick. The major benefit of OCT over other inspection techniques is that it has a long enough working distance to look through the bulk of thick optics while maintaining high resolution. This allows measurement of subsurface cracks not obtainable by any other known technique.

The time required to profile a damage sites is minimized by using an instrument which measures at high duty factor. We demonstrated a commercial instrument that utilized scanning rhombic prisms with a 90 percent duty factor providing 300 axial line-scans per second. The raw measurements were refined by applying a simple algorithm using the characteristic shape of the OCT signal. The refined measurements result in a profile which can be plotted with any chosen viewing angle. The inherently three dimensional nature of the OCT data is as accurate or in many respects superior to traditional microscopy.

OCT is still a rapidly developing technique. The total time to acquire and process the OCT data presented in this work is 4 to 6 hours per damage site. Other instruments in development may greatly reduce the scan time to the point where data could be taken in situ and in real-time. ${ }^{8}$ Measurement of the damage profile as it changes during $\mathrm{CO}_{2}$ laser mitigation is an example that would be highly valuable to optimization of the damage mitigation process.

References

1. Alan K. Burnham, Lloyd A. Hackel, Paul J. Wegner, Thomas G. Parham, Lawrence W. Hrubesh, Bernie M. Penetrante, Pamela K. Whitman, Stavros G. Demos, Joseph A. Menapace, Michael J. Runkel, Michael J. Fluss, Michael D. Feit, Michael H. Key, and Thomas A. Biesiada, "Improving 351-nm damage performance of largeaperture fused silica and DKDP optics", Proc. SPIE Int. Soc. Opt. Eng., 4679, 173, 2002

2. Mary A. Norton, Lawrence W. Hrubesh, Zhouling Wu, Eugene E. Donohue, Michael D. Feit, Mark R. Kozlowski, David Milam, Kurt P. Neeb, William A. Molander, Alexander M. Rubenchik, Walter D. Sell, and Paul J. Wegner, "Growth of laser-initiated damage in fused silica at 351 nm", Proc. SPIE Int. Soc. Opt. Eng., 4347, 468, 2001

3. Lawrence W. Hrubesh, Mary A. Norton, William A. Molander, Eugene E. Donohue, Stephen M. Maricle, Bernie Penetrante, Raymond M. Brusasco, Walter Grundler, Jim A. Butler, Jeff Carr, R. Hill, Leslie J. Summers, Michael D. Feit, Alexander M. Rubenchik, Michael H. Key, Paul J. Wegner, Alan K. Burnham, Lloyd A. Hackel, and Mark R. Kozlowski, "Methods for mitigating surface damage growth in NIF final optics", Proc. SPIE Int. Soc. Opt. Eng., 4679, 23, 2002

4. Isaac L. Bass, Gabriel M. Guss, and Richard P. Hackel, "Mitigation of laser damage growth in fused silica with a galvanometer scanned $\mathrm{CO}_{2}$ laser", Proc. SPIE Int. Soc. Opt. Eng., 5991, 59910C, 2005

5. Stavros G. Demos, Mike Staggs, Kaoru Minoshima, and James Fujimoto, "Characterization of laser induced damage sites in optical components", Optics Express, 10, 25, 2002

6. $\mathrm{MCam}-3 \mathrm{D}$ fiber based profilometer manufactured by Novacam Technologies Inc., 277 Lakeshore Road, PointeClaire, QC, H9S 4L2 Canada

7. M. L. Dufour, G. Lamouche, S. Vergnole, B. Gauthier, C. Padioleau, M. Hewko, S. Levesque, and V. Bartulovic, "Surface inspection of hard to reach industrial parts using low-coherence interferometry", Proc. SPIE Int. Soc. Opt. Eng., 6343, 63431Z, 2006

8. Yuuki Watanabe, Kazuhiko Yamada, and Manabu Sato, "Ultrahigh-speed axial-lateral parallel time domain optical coherence tomography”, Proc. SPIE Int. Soc. Opt. Eng., 6429, 642922, 2007 